\title{
Direct and inverse theorems on the approximation of almost periodic functions in Besicovitch-Stepanets spaces
}

\author{
Serdyuk A.S., Shidlich A.L. ${ }^{凶}$

\begin{abstract}
Direct and inverse approximation theorems are proved in the Besicovitch-Stepanets spaces $B \mathcal{S}^{p}$
Key words and phrases: direct approximation theorem, inverse approximation theorem, Jackson
\end{abstract} \\ of almost periodic functions in terms of the best approximations of functions and their generalized \\ moduli of smoothness. \\ type inequality, generalized module of smoothness.
}

Institute of Mathematics, National Academy of Sciences of Ukraine, 3 Tereschenkivska str., 01024, Kyiv, Ukraine

$凶$ Corresponding author

E-mail: sanatolii@ukr.net (Serdyuk A.S.), shidlich@gmail.com (Shidlich A.L.)

\section{Introduction}

In the paper, in terms of the best approximations and generalized moduli of smoothness, direct and inverse approximation theorems are established for Besicovitch almost periodic functions, the sequences of Fourier exponents of which have a single boundary point in infinity, and the sums of the $p$ th degrees of absolute values of the Fourier coefficients are finite. Study of direct and inverse approximation theorems originates in the well-known papers of D. Jackson [18] and S.N. Bernstein [8]. Such theorems establish connections between the difference-differential properties of the function that is approximated and the value of the error of its approximation by various methods (see, e.g. the monographs $[13,20,29,33])$. In 1962, N.P. Korneichuk [19] proved Jackson inequality in the uniform metric with the least (exact) constant. In [14], N.I. Chernykh showed that for an arbitrary $2 \pi$-periodic square-summable non-constant function $f\left(f \in L_{2}\right)$,

$$
E_{n}(f)_{2}<2^{-1 / 2} \omega(f, \pi / n)_{2}, \quad n \in \mathbb{N},
$$

where $E_{n}(f)_{2}$ is the best mean-square approximation of the function $f$ by trigonometric polynomials of order $n-1$, and $\omega(f, t)_{2}$ is the modulus of continuity (modulus of smoothness of the first order) of $f$ in the space $L_{2}$. In [15], the unimprovable Jackson-type inequalities with averaged moduli of smoothness with some weight functions were established.

In spaces of almost periodic functions, direct approximation theorems were established in the papers $[6,11,23,24]$, etc. In particular, in [23], an analogue of the inequality (1) was obtained for Besicovitch almost periodic functions of the order 2 ( $B^{2}$-a.p. functions). In [24] and [6], Jackson type inequalities were obtained with moduli of smoothness of $B^{2}$-a.p. functions of arbitrary positive integer order and with generalized moduli of smoothness, respectively. 
In this paper, we consider the spaces $B \mathcal{S}^{p}$ of all functions that are Besicovitch almost periodic of order 1 ( $B$-a.p. functions) for which the sums of the $p$ th degrees of absolute values of their Fourier coefficients are finite, $1 \leq p<\infty$. The norm of a function in the spaces $B \mathcal{S}^{p}$ is defined as the usual norm of a sequence of its Fourier coefficients in the space of numerical sequences $l_{p}$.

In the case when the $2 \pi$-periodic Lebesgue summable functions were considered instead of the $B$-a.p. functions, similar spaces were studied in the papers of A.I. Stepanets and his followers, and they were denoted by $\mathcal{S}^{p}[3,25-28,35,38],[29$, Ch. 11], etc. In [28], direct and inverse theorems for the approximation of functions from the spaces $\mathcal{S}^{p}$ were proved in terms of their best approximations by trigonometric polynomials and moduli of smoothness of arbitrary positive orders. In [3], exact Jackson-type inequalities in the spaces $\mathcal{S}^{p}$ were obtained in terms of the best approximations of functions and the averaged values of their generalized moduli of smoothness as well as the exact values were found for widths of classes of $2 \pi$-periodic functions defined by certain conditions on the averaged values of their generalized moduli of smoothness.

The spaces $B \mathcal{S}^{p}$ are a natural generalization of both the spaces $\mathcal{S}^{p}$ (since $\mathcal{S}^{p} \subset B \mathcal{S}^{p}$ ) and spaces $B^{2}$-a.p. functions (since the sets of $B^{2}$-a.p. functions coincide with the sets $B \mathcal{S}^{2}$ ). Therefore, it is of interest to obtain direct and inverse theorems on the approximation of functions from the spaces $B \mathcal{S}^{p}$ in terms of their best approximations and generalized moduli of smoothness.

\section{Preliminaries}

Let $B^{s}, 1 \leq s<\infty$, be the space of all functions Lebesgue summable with the sth degrees in each finite interval of the real axis, in which the distance is defined by the equality

$$
D_{B^{s}}(f, g)=\left(\varlimsup_{T \rightarrow \infty} \frac{1}{2 T} \int_{-T}^{T}|f(x)-g(x)|^{s} d x\right)^{1 / s} .
$$

Further, let $\mathcal{T}$ be the set of all trigonometric sums of the form $\tau_{N}(x)=\sum_{k=1}^{N} a_{k} e^{i \lambda_{k} x}, N \in \mathbb{N}$, where $\lambda_{k} \in \mathbb{R}$ and $a_{k} \in \mathbb{C}$ are arbitrary real and complex numbers, respectively.

An arbitrary function $f$ is called a Besicovitch almost periodic function of order $s$ (or $B^{s}$-a.p. function) and is denoted by $f \in B^{s}$-a.p. [22, Ch. 5, §10], [9, Ch. 2, §7], if there exists a sequence of trigonometric sums $\tau_{1}, \tau_{2}, \ldots$ from the set $\mathcal{T}$ such that $\lim _{N \rightarrow \infty} D_{B^{s}}\left(f, \tau_{N}\right)=0$.

If $s_{1} \geq s_{2} \geq 1$, then (see, e.g. [11,12]) $B^{s_{1}}$-a.p. $\subset B^{s_{2}}$-a.p. $\subset B$-a.p., where $B$-a.p. $:=B^{1}$-a.p.. For any $B$-a.p. function $f$, there exists the average value

$$
M\{f\}=\lim _{T \rightarrow \infty} \frac{1}{T} \int_{0}^{T} f(x) d x .
$$

The value of the function $M\left\{f(\cdot) e^{-i \lambda \cdot}\right\}, \lambda \in \mathbb{R}$, can be nonzero at most on a countable set. As a result of numbering the values of this set in an arbitrary order, we obtain a set $\mathcal{S}(f)=\left\{\lambda_{k}\right\}_{k \in \mathbb{N}}$ of Fourier exponents, which is called the spectrum of the function $f$. The numbers $A_{\lambda_{k}}=$ $A_{\lambda_{k}}(f)=M\left\{f(\cdot) e^{-i \lambda_{k} \cdot}\right\}$ are called the Fourier coefficients of the function $f$. To each function $f \in B$-a.p. with spectrum $\mathcal{S}(f)$ there corresponds a Fourier series of the form $\sum_{k} A_{\lambda_{k}} e^{i \lambda_{k} x}$. If, in addition, $f \in B^{2}$-a.p., then the Parseval equality holds (see, e.g. [9, Ch. 2, §9])

$$
M\left\{|f|^{2}\right\}=\sum_{k \in \mathbb{N}}\left|A_{\lambda_{k}}\right|^{2} .
$$


Developing the ideas of A.I. Stepanets [27], for a fixed $1 \leq p<\infty$ we consider the spaces of all functions $f \in B$-a.p., for which the following quantity is finite

$$
\|f\|_{p}:=\|f\|_{B \mathcal{S}^{p}}=\left\|\left\{A_{\lambda_{k}}(f)\right\}_{k \in \mathbb{N}}\right\|_{l_{p}(\mathbb{N})}=\left(\sum_{k \in \mathbb{N}}\left|A_{\lambda_{k}}(f)\right|^{p}\right)^{1 / p} .
$$

We denote these spaces by $B \mathcal{S}^{p}$ and call them Besicovitch-Stepanets spaces. By definition, $B$-a.p. functions are considered identical in $B \mathcal{S}^{p}$ if they have the same Fourier series.

Further, we will consider only those almost periodic functions from the spaces $B \mathcal{S}^{p}$, the sequences of Fourier exponents of which have a single limit point at infinity. For such functions $f$, the Fourier series are written in the symmetric form

$$
S[f](x)=\sum_{k \in \mathbb{Z}} A_{k} e^{i \lambda_{k} x}, \quad A_{k}=A_{k}(f)=M\left\{f(\cdot) e^{-i \lambda_{k} \cdot}\right\},
$$

where $\lambda_{0}:=0, \lambda_{-k}=-\lambda_{k},\left|A_{k}\right|+\left|A_{-k}\right|>0$, and $\lambda_{k+1}>\lambda_{k}>0$ for $k>0$.

By $G_{\lambda_{n}}$ we denote the set of all $B$-a.p. functions, whose Fourier exponents belong to the interval $\left(-\lambda_{n}, \lambda_{n}\right)$, and define the value of the best approximation by the equality

$$
E_{\lambda_{n}}(f)_{p}=E_{\lambda_{n}}(f)_{B \mathcal{S}^{p}}=\inf _{g \in G_{\lambda_{n}}}\|f-g\|_{p}
$$

Let $\Phi$ be the set of all continuous bounded nonnegative pair functions $\varphi(t)$ such that $\varphi(0)=0$ and the Lebesgue measure of the set $\{t \in \mathbb{R}: \varphi(t)=0\}$ is equal to zero. For an arbitrary fixed $\varphi \in \Phi$, consider the generalized modulus of smoothness of the function $f \in B \mathcal{S}^{p}$

$$
\omega_{\varphi}(f, \delta)_{p}:=\omega_{\varphi}(f, \delta)_{B \mathcal{S}^{p}}=\sup _{|h| \leq \delta}\left(\sum_{k \in \mathbb{Z}} \varphi^{p}\left(\lambda_{k} h\right)\left|A_{k}(f)\right|^{p}\right)^{1 / p}, \quad \delta \geq 0 .
$$

Let $\mathcal{M}=\left\{\mu_{j}\right\}_{j=0}^{m}$ be a nonzero collection of complex numbers such that $\sum_{j=0}^{m} \mu_{k}=0$. We associate the collection $\mathcal{M}$ with the difference operator $\Delta_{h}^{\mathcal{M}} f(t)=\sum_{j=0}^{m} \mu_{j} f(t-j h)$ and the modulus of smoothness $\omega_{\mathcal{M}}(f, \delta)_{p}:=\sup _{|h| \leq \delta}\left\|\Delta_{h}^{\mathcal{M}} f\right\|_{p}$. Note that the collection

$$
\mathcal{M}(m)=\left\{\mu_{j}=(-1)^{j}\left(\begin{array}{c}
m \\
j
\end{array}\right), j=0,1, \ldots, m\right\}, \quad m \in \mathbb{N},
$$

corresponds to the classical modulus of smoothness of order $m: \omega_{\mathcal{M}(m)}(f, \delta)_{p}=\omega_{m}(f, \delta)_{p}$.

For any $k \in \mathbb{Z}$, the Fourier coefficients of the function $\Delta_{h}^{M} f$ satisfy the equality

$$
\left|A_{k}\left(\Delta_{h}^{\mathcal{M}} f\right)\right|=\left|A_{k}(f)\right|\left|\sum_{j=0}^{m} \mu_{j} e^{-i \lambda_{k} j h}\right| .
$$

Therefore, for $\varphi_{\mathcal{M}}(t)=\left|\sum_{j=0}^{m} \mu_{j} e^{-i j t}\right|$ we have $\omega_{\varphi_{\mathcal{M}}}(f, \delta)_{p}=\omega_{\mathcal{M}}(f, \delta)_{p}$. In particular, for $\varphi_{m}(t)=2^{m}|\sin (t / 2)|^{m}=2^{m / 2}(1-\cos t)^{m / 2}, m \in \mathbb{N}$, we have $\omega_{\varphi_{m}}(f, \delta)_{p}=\omega_{m}(f, \delta)_{p}$.

In the general case, such modules were studied in $[1,3,5,6,10,21,36,37]$, etc.

\section{Main results}

\subsection{Jackson type inequalities}

In this subsection, direct approximation theorems are established in the space $B \mathcal{S}^{p}$ in terms of best approximations and generalized moduli of smoothness. For functions $f \in B \mathcal{S}^{p}$ with 
the Fourier series of the form (3), we prove Jackson type inequalities of the kind

$$
E_{\lambda_{n}}(f)_{p} \leq K(\tau) \omega_{\varphi}\left(f, \frac{\tau}{\lambda_{n}}\right)_{p}{ }^{\prime} \quad \tau>0, \quad 1 \leq p<\infty, \quad n \in \mathbb{N},
$$

and consider the problem of the least constant in these inequalities for fixed values of the parameters $n, \varphi, \tau$ and $p$. In particular, we study the quantity

$$
K_{n, \varphi, p}(\tau)=\sup \left\{\frac{E_{\lambda_{n}}(f)_{p}}{\omega_{\varphi}\left(f, \tau / \lambda_{n}\right)_{p}}: f \in B \mathcal{S}^{p}\right\}
$$

Here and below, we assume that $0 / 0=0$.

Let $V(\tau), \tau>0$, be a set of bounded nondecreasing functions $v$ that differ from a constant on $[0, \tau]$.

Theorem 1. Assume that the function $f \in B \mathcal{S}^{p}, 1 \leq p<\infty$, has the Fourier series of the form (3). Then for any $\tau>0, n \in \mathbb{N}$ and $\varphi \in \Phi$ the following inequality holds

$$
E_{\lambda_{n}}(f)_{p} \leq C_{n, \varphi, p}(\tau) \omega_{\varphi}\left(f, \frac{\tau}{\lambda_{n}}\right)_{p}{ }^{\prime}
$$

where

and

$$
C_{n, \varphi, p}(\tau):=\left(\inf _{v \in M(\tau)} \frac{v(\tau)-v(0)}{I_{n, \varphi, p}(\tau, v)}\right)^{1 / p}
$$

$$
I_{n, \varphi, p}(\tau, v):=\inf _{k \in \mathbb{N}, k \geq n} \int_{0}^{\tau} \varphi^{p}\left(\frac{\lambda_{k} t}{\lambda_{n}}\right) d v(t) .
$$

Futhermore, there exists a function $v_{*} \in V(\tau)$ that realizes the greatest lower bound in (6). Inequality (5) is unimprovable on the set of all functions $f \in B \mathcal{S}^{p}$ with the Fourier series of the form (3) in the sense that for any $\varphi \in \Phi$ and $n \in \mathbb{N}$ the following equality is true

$$
C_{n, \varphi, p}(\tau)=K_{n, \varphi, p}(\tau)
$$

In the spaces $L_{2}$ of $2 \pi$-periodic square-summable functions, for moduli of continuity, this result was obtained by A.G. Babenko [4]. In the spaces $\mathcal{S}^{p}$ of functions of one and several variables, this result for classical moduli of smoothness was obtained in [28] and [2], respectively, and for generalized moduli of smoothness, in [1] (for functions of one variable). In the proof of Theorem 1, we mainly use the ideas outlined in $[4,14,15,28]$, taking into account the peculiarities of the spaces $B \mathcal{S}^{p}$.

Proof. From relations (2) and (8), it follows that for any $f \in B \mathcal{S}^{p}$ with the Fourier series of the form (3), we have

$$
E_{\lambda_{n}}^{p}(f)_{p}=\left\|f-S_{n}(f)\right\|_{p}=\sum_{|k| \geq n}\left|A_{k}(f)\right|^{p},
$$

where $S_{n}(f):=\sum_{|k|<n} A_{k}(f) e^{i \lambda_{k} x}$.

For any $f \in B \mathcal{S}^{p}, \varphi \in \Phi$ and $h \in \mathbb{R}$, consider the sequence of numbers $\left\{\varphi\left(\lambda_{k} h\right) A_{k}(f)\right\}_{k \in \mathbb{Z}}$. If there exists a function $\Delta_{h}^{\varphi} f \in B$-a.p. such that for all $k \in \mathbb{Z}$

$$
A_{k}\left(\Delta_{h}^{\varphi} f\right)=\varphi\left(\lambda_{k} h\right) A_{k}(f),
$$

then denote by $\left\|\Delta_{h}^{\varphi} f\right\|_{p}$ the usual norm (2) of the function $\Delta_{h}^{\varphi} f$. If such a $B$-a.p function $\Delta_{h}^{\varphi} f$ does not exist, then to simplify notation we will also use the notation $\left\|\Delta_{h}^{\varphi} f\right\|_{p}$, meaning by it

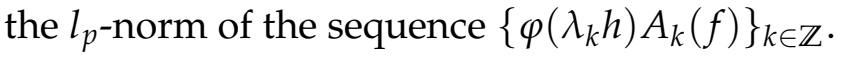


Taking into account (7), (8) and the parity of the function $\varphi$, we obtain

$$
\begin{aligned}
\left\|\Delta_{h}^{\varphi} f\right\|_{p}^{p} & =\sum_{k \in \mathbb{Z}} \varphi^{p}\left(\lambda_{k} h\right)\left|A_{k}(f)\right|^{p} \geq \sum_{|k| \geq n} \varphi^{p}\left(\lambda_{k} h\right)\left|A_{k}(f)\right|^{p} \\
& =\frac{I_{n, \varphi, p}(\tau, v)}{v(\tau)-v(0)} E_{\lambda_{n}}^{p}(f)_{p}+\sum_{|k| \geq n}\left|A_{k}(f)\right|^{p}\left(\varphi^{p}\left(\lambda_{k} h\right)-\frac{I_{n, \varphi, p}(\tau, v)}{v(\tau)-v(0)}\right),
\end{aligned}
$$

where the quantity $I_{n, \varphi, p}(\tau, v)$ is defined by (7). Hence, for any $t \in[0, \tau]$ we find

$$
E_{\lambda_{n}}^{p}(f)_{p} \leq \frac{v(\tau)-v(0)}{I_{n, \varphi, p}(\tau, v)}\left(\left\|\Delta_{t / \lambda_{n}}^{\varphi} f\right\|_{p}^{p}-\sum_{|k| \geq n}\left|A_{k}(f)\right|^{p}\left(\varphi^{p}\left(\frac{\lambda_{k} t}{\lambda_{n}}\right)-\frac{I_{n, \varphi, p}(\tau, v)}{v(\tau)-v(0)}\right)\right) .
$$

Since the both sides of inequality (10) are nonnegative and the series on its right-hand side is majorized on the entire real axis by the absolutely convergent series $C(\varphi) \sum_{|k| \geq n}\left|A_{k}(f)\right|^{p}$, where $C(\varphi)=\max _{t \in \mathbb{R}} \varphi(t)$, then integrating this inequality with respect to $\mathrm{d} v(t)$ from 0 to $\tau$, we get

$$
\begin{aligned}
E_{\lambda_{n}}^{p}(f)_{p}(v(\tau)-v(0)) \leq & \frac{v(\tau)-v(0)}{I_{n, \varphi, p}(\tau, v)}\left(\int_{0}^{\tau}\left\|\Delta_{t / \lambda_{n}}^{\varphi} f\right\|_{p}^{p} d v(t)\right. \\
& \left.\quad-\sum_{|k| \geq n}\left|A_{k}(f)\right|^{p}\left(\int_{0}^{\tau} \varphi^{p}\left(\frac{\lambda_{k} t}{\lambda_{n}}\right) d v(t)-I_{n, \varphi, p}(\tau, v)\right)\right)
\end{aligned}
$$

By virtue of (7), we have

$$
\int_{0}^{\tau} \varphi^{p}\left(\frac{\lambda_{k} t}{\lambda_{n}}\right) d v(t)-I_{n, \varphi, p}(\tau, v) \geq 0 .
$$

Therefore, for any function $v \in V(\tau)$, we have

$$
E_{\lambda_{n}}^{p}(f)_{p} \leq \frac{1}{I_{n, \varphi, p}(\tau, v)} \int_{0}^{\tau}\left\|\Delta_{t / \lambda_{n}}^{\varphi} f\right\|_{p}^{p} d v(t) \leq \frac{1}{I_{n, \varphi, p}(\tau, v)} \int_{0}^{\tau} \omega_{\varphi}^{p}\left(f, \frac{t}{\lambda_{n}}\right) d v(t) .
$$

Hence we immediately get (5) and the estimate

$$
K_{n, \varphi, p}^{p}(\tau) \leq \inf _{v \in V(\tau)} \frac{v(\tau)-v(0)}{I_{n, \varphi, p}(\tau, v)}=C_{n, \varphi, p}^{p}(\tau) .
$$

It remains to show that in relation (12) there is in fact equality.

By virtue of (4) and (8), we have

$$
K_{n, \varphi, p}^{p}(\tau)=\sup _{f \in B \mathcal{S}^{p}} \frac{\sum_{|k| \geq n}\left|A_{k}(f)\right|^{p}}{\sup _{|h| \leq \tau} \sum_{|k| \geq n} \varphi^{p}\left(\lambda_{k} h / \lambda_{n}\right)\left|A_{k}(f)\right|^{p}} .
$$

In (13), it is sufficient to consider the supremum over all functions $f \in B \mathcal{S}^{p}$, such that

$$
\sum_{|k| \geq n}\left|A_{k}(f)\right|^{p} \leq 1
$$

Then, taking into account the parity of the function $\varphi$, we obtain

$$
K_{n, \varphi, p}^{-p}(\tau) \leq J_{n, \varphi, p}(\tau):=\inf _{w \in W_{n, \varphi, p}}\|w\|_{C_{[0, \tau]}}
$$

where

$$
W_{n, \varphi, p}:=\left\{\omega(u)=\sum_{j=n}^{\infty} \varrho_{j} \varphi^{p}\left(\frac{\lambda_{j} u}{\lambda_{n}}\right): \varrho_{j} \geq 0, \sum_{j=n}^{\infty} \varrho_{j}=1\right\} .
$$

Further, we use the duality relation in the space $C_{[a, b]}$, which we formulate as the following statement (see, e.g. [20, Ch. 1.4]). 
Proposition 1 ([20, Ch. 1.4]). If $F$ is a convex set in the space $C_{[a, b]}$, then for any $x \in C_{[a, b]}$

$$
\inf _{u \in F}\|x-u\|_{C_{[a, b]}}=\sup _{\substack{b \\ V \\ a}}\left(\int_{a}^{b} x(t) d g(t)-\sup _{u \in F} \int_{a}^{b} u(t) d g(t)\right) .
$$

For $x \in C_{[a, b]} \backslash \bar{F}$, where $\bar{F}$ is the closure of a set $F$, there exists a function $g$ with variation equal to 1 on $[a, b]$ that realizes the least upper bound in (16).

It is easy to see that the set $W_{n, \alpha, p}$ is a convex subset of the space $C_{[0, \tau]}$. Therefore, setting $a=0, b=\tau, x(t) \equiv 0, u(t)=w(t) \in W_{n, \alpha, p}, F=W_{n, \alpha, p}$, from relation (16) we get

$$
\begin{aligned}
J_{n, \varphi, p}(\tau) & =\inf _{\substack{w \in W_{n, \varphi, p}\\
}}\|0-w\|_{C_{[0, \tau]}} \\
& =\sup _{\substack{\tau \\
V \\
0}}\left(0-\sup _{\substack{w \in W_{n, \varphi, p} \leq 1\\
}} \int_{0}^{\tau} w(t) d g(t)\right)=\sup _{\substack{\tau \\
V \\
0}} \inf _{\substack{(g) \leq 1 \\
w \in W_{n, \varphi, p}}} \int_{0}^{\tau} w(t) d g(t) .
\end{aligned}
$$

Furthermore, according to Proposition 1, there exists a function $g_{*}(t)$, that realizes the least upper bound in (17) and such that $\underset{0}{V}\left(g_{*}\right)=1$. Every function $w \in W_{n, \alpha, p}$ is nonnegative. Therefore, it sufficient to take the supremum on the right-hand side of (17) over the set of nondecreasing functions $v(t)$ for which $v(\tau)-v(0) \leq 1$. For such functions, by virtue of $(7)$ and (15), the following equality

$$
\inf _{w \in W_{n, \alpha, p}} \int_{0}^{\tau} w(t) d v(t)=I_{n, \varphi, p}(\tau, v) .
$$

is true. This implies that there exists a function $v_{*} \in V(\tau)$ such that $v_{*}(\tau)-v_{*}(0)=1$ and

$$
I_{n, \varphi, p}\left(\tau, v_{*}\right)=\sup _{v \in V(\tau), \underset{0}{\tau}(v) \leq 1} I_{n, \varphi, p}(\tau, v)=J_{n, \varphi, p}(\tau) .
$$

From relations (14) and (18), we obtain the necessary estimate

$$
K_{n, \varphi, p}^{p}(\tau) \geq \frac{1}{J_{n, \varphi, p}(\tau)}=\frac{1}{I_{n, \varphi, p}\left(\tau, v_{*}\right)}=\frac{v_{*}(\tau)-v_{*}(0)}{I_{n, \varphi, p}\left(\tau, v_{*}\right)}=C_{n, \varphi, p}^{p}(\tau) .
$$

Consider an important special case when

$$
\varphi(t)=\varphi_{\alpha}(t)=2^{\alpha / 2}(1-\cos t)^{\alpha / 2}=2^{\alpha}|\sin (t / 2)|^{\alpha}, \quad \alpha>0 .
$$

In this case, we set $\omega_{\varphi_{\alpha}}(f, \delta)_{p}=: \omega_{\alpha}(f, \delta)_{p}$ and $K_{n, \varphi_{\alpha}, p}(\tau)=: K_{n, \alpha, p}(\tau)$. For the weight function $v_{1}(t)=1-\cos t$, we get the following assertion.

Corollary 1. For any function $f \in B \mathcal{S}^{p}, 1 \leq p<\infty$, with the Fourier series of the form (3), the following inequalities

$$
E_{\lambda_{n}}^{p}(f)_{p} \leq \frac{1}{2^{\alpha p / 2} I_{n}(\alpha p / 2)} \int_{0}^{\pi} \omega_{\alpha}^{p}\left(f, \frac{t}{\lambda_{n}}\right)_{p} \sin t d t, \quad n \in \mathbb{N}, \quad \alpha>0,
$$

hold, where

$$
I_{n}(s):=\inf _{k \in \mathbb{N}, k \geq n} \int_{0}^{\pi}\left(1-\cos \frac{\lambda_{k} t}{\lambda_{n}}\right)^{s} \sin t d t, \quad s>0, \quad n \in \mathbb{N}
$$


If, in addition $\alpha p / 2 \in \mathbb{N}$, then

$$
I_{n}\left(\frac{\alpha p}{2}\right)=\frac{2^{\alpha p / 2+1}}{\alpha p / 2+1}
$$

and inequality (19) cannot be improved for any $n \in \mathbb{N}$.

Proof. Inequality (19) follows from relation (11) with $\tau=\pi, \varphi(t)=\varphi_{\alpha}(t)$ and $v(t)=1-\cos t$, $t \in[0, \pi]$. In [28, relation (52)], it was shown that for any $\theta \geq 1$ and $s \in \mathbb{N}$ the following inequality

$$
\int_{0}^{\pi}(1-\cos \theta t)^{s} \sin t d t \geq \frac{2^{s+1}}{s+1}
$$

holds, which turns into equality for $\theta=1$. Therefore, setting $s=\alpha p / 2$ and $\theta=\lambda_{v} / \lambda_{n}$, $v=n, n+1, \ldots$, and the monotonicity of the sequence of Fourier exponents $\left\{\lambda_{k}\right\}_{k \in \mathbb{Z}}$, we see that for $\alpha p / 2 \in \mathbb{N}$, indeed, the equality (21) holds.

To prove that inequality (19) is unimprovable for $\alpha p / 2 \in \mathbb{N}$, it suffices to verify that the function

$$
f^{*}(x)=\gamma+\beta e^{-\lambda_{n} x}+\delta e^{\lambda_{n} x}
$$

where $\gamma, \beta$ and $\delta$ are arbitrary complex numbers, satisfies the equality

$$
E_{\lambda_{n}}^{p}\left(f^{*}\right)_{p}=\frac{\alpha p / 2+1}{2^{\alpha p+1}} \int_{0}^{\pi} \omega_{\alpha}^{p}\left(f^{*}, \frac{t}{\lambda_{n}}\right)_{p} \sin t d t, \quad n \in \mathbb{N}, \quad \alpha>0 .
$$

In this case, $E_{\lambda_{n}}^{p}\left(f^{*}\right)_{p}^{p}=|\beta|^{p}+|\delta|^{p}$, the function $\left\|\Delta_{t / \lambda_{n}}^{\varphi_{\alpha}} f^{*}\right\|_{p}^{p}=2^{\alpha p / 2}\left(|\beta|^{p}+|\delta|^{p}\right)(1-\cos t)^{\alpha p / 2}$ does not decrease with respect to $t$ on $[0, \pi]$. Therefore, $\omega_{\alpha}\left(f^{*}, t / \lambda_{n}\right)_{p}=\left\|\Delta_{t / \lambda_{n}}^{\varphi_{\alpha}} f^{*}\right\|_{p}$, and

$$
\begin{aligned}
\frac{2^{\alpha p+1}}{\alpha p / 2+1} E_{\lambda_{n}}^{p}\left(f^{*}\right)_{p}-\int_{0}^{\pi} \omega_{\alpha}^{p} & \left(f^{*}, \frac{t}{\lambda_{n}}\right)_{p} \sin t d t \\
& =\left(|\beta|^{p}+|\delta|^{p}\right)\left(\frac{2^{\alpha p+1}}{\alpha p / 2+1}-2^{\alpha p / 2} \int_{0}^{\pi}(1-\cos t)^{\alpha p ? 2} \sin t d t\right)=0 .
\end{aligned}
$$

If $v_{2}(t)=t$, then we obtain the following assertion.

Corollary 2. Assume that the function $f \in B \mathcal{S}^{p}, 1 \leq p<\infty$, has the Fourier series of the form (3) and the number $\alpha>0$ such that $\alpha p \geq 1$. Then for any $0<\tau \leq 3 \pi / 4$ and $n \in \mathbb{N}$,

$$
E_{\lambda_{n}}^{p}(f)_{p} \leq\left(\int_{0}^{\tau} \omega_{\alpha}^{p}\left(f, \frac{t}{\lambda_{n}}\right)_{p} d t\right) /\left(2^{\alpha p} \int_{0}^{\tau} \sin ^{\alpha p} \frac{t}{2} d t\right) .
$$

Equality in (24) holds for the function $f^{*}$ of the form (22).

Proof. From inequality (11), it follows that

$$
E_{\lambda_{n}}^{p}(f)_{p} \leq \frac{1}{\tilde{I}_{n}(\alpha p / 2)} \int_{0}^{\tau} \omega_{\alpha}^{p}\left(f, \frac{t}{\lambda_{n}}\right) d t
$$

where

$$
\tilde{I}_{n}(s):=\inf _{k \in \mathbb{N}, k \geq n} \int_{0}^{\tau}\left(1-\cos \frac{\lambda_{k} t}{\lambda_{n}}\right)^{s} d t, \quad s>0, \quad n \in \mathbb{N} .
$$


Consider the function

$$
F_{\beta}(x):=\frac{1}{x} \int_{0}^{x}|\sin t|^{\beta} d t .
$$

In [38], it is shown that for any $h \in(0,3 \pi / 4)$ and $\beta \geq 1$, the following relation

$$
\inf _{x \geq h / 2} F_{\beta}(x)=F_{\beta}(h / 2) \text {. }
$$

is true. Since for any $\theta \in \mathbb{R}$,

$$
\int_{0}^{\tau}(1-\cos \theta t)^{\beta / 2} d t=2^{\beta} \int_{0}^{\tau}\left|\sin \frac{\theta t}{2}\right|^{\beta} d t=2^{\beta} \tau F_{\beta}\left(\frac{\theta \tau}{2}\right),
$$

then setting $\theta=\lambda_{k} / \lambda_{n} \geq 1(k \geq n)$ and $\beta=\alpha p$, from (25) (with $\left.\tau \in(0,3 \pi / 4]\right)$ we obtain

$$
\begin{aligned}
\tilde{I}_{n}\left(\frac{\alpha p}{2}\right) & =\inf _{k \in \mathbb{N}, k \geq n} \int_{0}^{\tau}\left(1-\cos \frac{\lambda_{k} t}{\lambda_{n}}\right)^{\alpha p / 2} d t \\
& =2^{\alpha p} \inf _{k \in \mathbb{N}, k \geq n} \int_{0}^{\tau}\left|\sin \frac{\lambda_{k} t}{2 \lambda_{n}}\right|^{\alpha p} d t=2^{\alpha p} \int_{0}^{\tau} \sin ^{\alpha p} \frac{t}{2} d t .
\end{aligned}
$$

For the functions $f^{*}$ of the form (22), the equality

$$
E_{\lambda_{n}}^{p}\left(f^{*}\right)_{p}=\left(\int_{0}^{\tau} \omega_{\alpha}^{p}\left(f^{*}, \frac{t}{\lambda_{n}}\right)_{p} d t\right) /\left(2^{\alpha p} \int_{0}^{\tau} \sin ^{\alpha p} \frac{t}{2} d t\right) .
$$

is verified similarly to the proof of equality (23).

In the following assertion, we give the upper estimates for the least constants $K_{n, \alpha, p}(\tau)$ in Jackson type inequalities with the moduli of smoothness $\omega_{\alpha}(f, \cdot)_{p}$ and $\tau=\pi$. These estimates do not depend on $n$ and are unimprovable in several important cases.

Corollary 3. For any $n \in \mathbb{N}$ and $\alpha>0$, the following inequalities

$$
K_{n, \alpha, p}^{p}(\pi) \leq \frac{1}{2^{\alpha p / 2-1} I_{n}(\alpha p / 2)} \leq \frac{\alpha p / 2+1}{2^{\alpha p}+2^{\alpha p / 2-1}(\alpha p / 2+1) \sigma(\alpha p / 2)},
$$

are true, where the quantities $I_{n}(s), s>0$, are defined by (20), and

$$
\sigma(s):=-\sum_{\alpha=[s / 2]+1}^{\infty}\left(\begin{array}{c}
s \\
2 \alpha
\end{array}\right) \frac{1}{2^{2 \alpha-1}}\left(\frac{1-(-1)^{[s]}}{2}\left(\begin{array}{c}
2 \alpha \\
\alpha
\end{array}\right)-\sum_{j=0}^{\alpha-1}\left(\begin{array}{c}
2 \alpha \\
j
\end{array}\right) \frac{2}{2(\alpha-j)^{2}-1}\right),
$$

here $[s]$ is the integer part of the number s. If $\alpha p / 2 \in \mathbb{N}$, then $\sigma(\alpha p / 2)=0$ and

$$
K_{n, \alpha, p}^{p}(\pi) \leq \frac{\alpha p / 2+1}{2^{\alpha p}}, \quad \frac{\alpha p}{2} \in \mathbb{N}, \quad n \in \mathbb{N} .
$$

Proof. The first inequality in (26) and inequality (27) follow from Corollary 1. The second inequality in (26) follows from the relation

$$
I_{n}\left(\frac{\alpha p}{2}\right) \geq \frac{2^{\alpha p / 2+1}}{\alpha p / 2+1}+\sigma\left(\frac{\alpha p}{2}\right), \quad n \in \mathbb{N}, \quad \alpha>0,
$$

which is a consequence of the inequality (see [28])

$$
\int_{0}^{\pi}(1-\cos \theta t)^{s} \sin t d t \geq \frac{2^{s+1}}{s+1}+\sigma(s), \quad \theta \geq 1, \quad s>0 .
$$


The following assertion establishes the uniform boundedness of the constants $K_{n, \alpha, p}(\pi)$ with respect to the parameters $n \in \mathbb{N}$ and $1 \leq p<\infty$.

Corollary 4. Assume that the function $f \in B \mathcal{S}^{p}, 1 \leq p<\infty$, has the Fourier series of the form (3) and $\left\|f-A_{0}(f)\right\|_{p} \neq 0$. Then for any $n \in \mathbb{N}$ and $\alpha>0$,

$$
E_{\lambda_{n}}(f)_{p}<\frac{(4 / 3)^{1 / p}}{2^{\alpha / 2}} \omega_{\alpha}\left(f, \frac{\pi}{\lambda_{n}}\right)_{p} \leq \frac{4}{3 \cdot 2^{\alpha / 2}} \omega_{\alpha}\left(f, \frac{\pi}{\lambda_{n}}\right)_{p}
$$

Furthermore, in the case where $\alpha=m \in \mathbb{N}$, the following more accurate estimate

$$
E_{\lambda_{n}}(f)_{p}<\frac{4-2 \sqrt{2}}{2^{m / 2}} \omega_{m}\left(f, \frac{\pi}{\lambda_{n}}\right)_{p}
$$

holds.

Proof. It was shown in [28] that $I_{n}(s) \geq 2$ when $s \geq 1$ and $I_{n}(s) \geq 1+2^{s-1}$ when $s \in(0,1)$. Combining these two estimates and (26), we get (28).

Relation (29) follows from the estimate $I_{n}(m p / 2) \geq 1+1 / \sqrt{2}$, which is a consequence of the above estimates for the value of $I_{n}(s)$ in the case when $m \in \mathbb{N}$ and $1 \leq p<\infty$ (see [28]).

As noted above, for $p=2$ the sets $B \mathcal{S}^{p}=B \mathcal{S}^{2}$ coincide with the sets of $B^{2}$-a.p. functions. Given the importance of this case, we give the formulation of the corresponding statements for the classical modulus of smoothness $\omega_{m}$, which follow from the Corollary 1.

Corollary 5. For any $B^{2}$-a.p. function $f$ having the Fourier series of the form (3), the inequalities

$$
E_{\lambda_{n}}^{2}(f)_{2} \leq \frac{m+1}{2^{2 m+1}} \int_{0}^{\pi} \omega_{m}^{2}\left(f, \frac{t}{\lambda_{n}}\right)_{2} \sin t d t, \quad m, n \in \mathbb{N} .
$$

hold. These inequalities can not be improved for any $m$ and $n \in \mathbb{N}$.

Corollary 6. For any $B^{2}$-a.p. function $f$ with the Fourier series of the form (3) such that $\left\|f-A_{0}(f)\right\|_{p} \neq 0$, the following inequalities

$$
E_{\lambda_{n}}(f)_{2}<\frac{\sqrt{m+1}}{2^{m}} \omega_{m}\left(f, \frac{\pi}{\lambda_{n}}\right)_{2^{\prime}} \quad m, n \in \mathbb{N}
$$

hold.

Inequalities (30) and (31) complement the results obtained in $[5,23,24]$, etc., for the $B^{2}$-a.p. functions. In the spaces $\mathcal{S}^{p}$ of functions of one and several variables, Theorem 1 and Corollaries 1, 3 and 4 were proved in [28] and [2], respectively. In the spaces $L_{2}$, for classical moduli of smoothness inequality (19) was proved by N.I. Chernykh [15]. The inequalities of this type were also investigated in $[3,5,6,17,25,31,32,35-38]$, etc.

\section{Inverse approximation theorems}

Theorem 2. Assume that the function $f \in B \mathcal{S}^{p}, 1 \leq p<\infty$, has the Fourier series of the form (3), the function $\varphi \in \Phi$ does not decrease on $[0, \tau], \tau>0$, and $\varphi(\tau)=\max \{\varphi(t): t \in \mathbb{R}\}$. Then for any $n \in \mathbb{N}$, the following inequality

$$
\omega_{\varphi}^{p}\left(f, \frac{\tau}{\lambda_{n}}\right) \leq \sum_{v=1}^{n}\left(\varphi^{p}\left(\frac{\tau \lambda_{v}}{\lambda_{n}}\right)-\varphi^{p}\left(\frac{\tau \lambda_{v-1}}{\lambda_{n}}\right)\right) E_{\lambda_{v}}^{p}(f)_{p}
$$

holds. 
Proof. Let us use the scheme of the proof from $[1,28]$, taking into account the peculiarities of the spaces $B \mathcal{S}^{p}$. As above, for any $f \in B \mathcal{S}^{p}, \varphi \in \Phi$ and $h \in \mathbb{R}$, we denote by $\left\|\Delta_{h}^{\varphi} f\right\|_{p}$ the usual norm (2) of the function $\Delta_{h}^{\varphi} f$ satisfying relation (9) (if such a function $\Delta_{h}^{\varphi} f \in B$-a.p. exists) or the $l_{p}$-norm of the sequence $\left\{\varphi\left(\lambda_{k} h\right) A_{k}(f)\right\}_{k \in \mathbb{Z}}$ if such a function $\Delta_{h}^{\varphi} f \in B$-a.p. does not exist. We have

$$
\left\|\Delta_{h}^{\varphi} f\right\|_{p}^{p}=\sum_{k \in \mathbb{Z}} \varphi^{p}\left(\lambda_{k} h\right)\left|A_{k}(f)\right|^{p}=\sum_{|k|<n} \varphi^{p}\left(\lambda_{k} h\right)\left|A_{k}(f)\right|^{p}+\sum_{|k| \geq n} \varphi^{p}\left(\lambda_{k} h\right)\left|A_{k}(f)\right|^{p} .
$$

It is clear that the second term on the right-hand side (33) does not exceed the value

$$
\varphi^{p}(\tau) \sum_{|k| \geq n}\left|A_{k}(f)\right|^{p}=\varphi^{p}(\tau) E_{\lambda_{n}}^{p}(f)_{p}
$$

and due to the parity and non-decreasing function $\varphi$ on the interval $[0, \tau]$, for $|h| \leq \tau / \lambda_{n}$

$$
\sum_{|k|<n} \varphi^{p}\left(\lambda_{k} h\right)\left|A_{k}(f)\right|^{p} \leq \sum_{v=1}^{n-1} \varphi^{p}\left(\frac{\tau \lambda_{v}}{\lambda_{n}}\right)\left(\left|A_{-v}(f)\right|^{p}+\left|A_{v}(f)\right|^{p}\right) .
$$

Therefore, in view of the monotonicity of the sequence of Fourier exponents $\left\{\lambda_{k}\right\}_{k \in \mathbb{Z}}$, we obtain

$$
\left\|\Delta_{h}^{\varphi} f\right\|_{p}^{p} \leq \varphi^{p}(\tau) E_{\lambda_{n}}^{p}(f)_{p}+\sum_{v=1}^{n-1} \varphi^{p}\left(\frac{\tau \lambda_{v}}{\lambda_{n}}\right) H_{v}^{p}(f)
$$

where $H_{v}^{p}(f)=\left(\left|A_{-v}(f)\right|^{p}+\left|A_{v}(f)\right|^{p}\right)$. Further, we use the following assertion from [28].

Lemma 1 ([28]). Assume that the numerical series $\sum_{v=1}^{\infty} c_{v}$ is convergent. Then for any sequence $\beta_{v}, v \in \mathbb{N}$, the following equality

$$
\sum_{v=N_{1}}^{N_{2}} \beta_{v} c_{v}=\beta_{a} \sum_{v=N_{1}}^{\infty} c_{v}+\sum_{v=N_{1}+1}^{N_{2}}\left(\beta_{v}-\beta_{v-1}\right) \sum_{i=v}^{\infty} c_{i}-\beta_{N_{2}} \sum_{v=N_{2}+1}^{\infty} c_{v}
$$

holds for all positive integers $N_{1}$ and $N_{2}, N_{1} \leq N_{2}$.

Setting $N_{1}=1, N_{2}=n-1, \beta_{v}=\varphi^{p}\left(\tau \lambda_{v} / \lambda_{n}\right)$ and $c_{v}=H_{v}^{p}(f)$ in (35), taking into account (8), we get

$$
\begin{aligned}
\sum_{v=1}^{n-1} \varphi^{p}\left(\frac{\tau \lambda_{v}}{\lambda_{n}}\right) H_{v}^{p}(f)= & \varphi^{p}\left(\frac{\tau \lambda_{1}}{\lambda_{n}}\right) \sum_{v=1}^{\infty} H_{v}^{p}(f)+\sum_{v=2}^{n-1}\left(\varphi^{p}\left(\frac{\tau \lambda_{v}}{\lambda_{n}}\right)-\varphi^{p}\left(\frac{\tau \lambda_{v-1}}{\lambda_{n}}\right)\right) \sum_{i=v}^{\infty} H_{i}^{p}(f) \\
& -\varphi^{p}\left(\frac{\tau \lambda_{n-1}}{\lambda_{n}}\right) \sum_{v=n}^{\infty} H_{v}^{p}(f) \\
= & \sum_{v=1}^{n-1}\left(\varphi^{p}\left(\frac{\tau \lambda_{v}}{\lambda_{n}}\right)-\varphi^{p}\left(\frac{\tau \lambda_{v-1}}{\lambda_{n}}\right)\right) E_{\lambda_{v}}^{p}(f)_{p}-\varphi^{p}\left(\frac{\tau \lambda_{n-1}}{\lambda_{n}}\right) E_{\lambda_{n}}^{p}(f)_{p} .
\end{aligned}
$$

By virtue of (34) and (36), we get

$$
\begin{aligned}
\left\|\Delta_{h}^{\varphi} f\right\|_{p}^{p} & \leq \sum_{v=1}^{n-1}\left(\varphi^{p}\left(\frac{\tau \lambda_{v}}{\lambda_{n}}\right)-\varphi^{p}\left(\frac{\tau \lambda_{v-1}}{\lambda_{n}}\right)\right) E_{\lambda_{v}}^{p}(f)_{p}-\varphi^{p}\left(\frac{\tau \lambda_{n-1}}{\lambda_{n}}\right) E_{\lambda_{n}}^{p}(f)_{p}+\varphi^{p}(\tau) E_{\lambda_{n}}^{p}(f)_{p} \\
& =\sum_{v=1}^{n}\left(\varphi^{p}\left(\frac{\tau \lambda_{v}}{\lambda_{n}}\right)-\varphi^{p}\left(\frac{\tau \lambda_{v-1}}{\lambda_{n}}\right)\right) E_{\lambda_{v}}^{p}(f)_{p},
\end{aligned}
$$

which yields (32). 
Consider the case $\varphi(t)=\varphi_{\alpha}(t)=2^{\alpha}|\sin (t / 2)|^{\alpha}, \alpha>0$. In this case, the function $\varphi$ satisfies the conditions of Theorem 2 with $\tau=\pi$. If $r=\alpha p \geq 1$, then using the inequality

$$
x^{r}-y^{r} \leq r x^{r-1}(x-y), \quad x>0, y>0
$$

(see, e.g. [16, Ch. 1]), and ordinary trigonometric transformations for $v=1,2, \ldots, n$, we get

$$
\begin{aligned}
\varphi^{p}\left(\frac{\tau \lambda_{v}}{\lambda_{n}}\right)-\varphi^{p}\left(\frac{\tau \lambda_{v-1}}{\lambda_{n}}\right) & =2^{\alpha p}\left(\left|\sin \frac{\pi \lambda_{v}}{\lambda_{n}}\right|^{\alpha p}-\left|\sin \frac{\pi \lambda_{v-1}}{\lambda_{n}}\right|^{\alpha p}\right) \\
& \leq 2^{\alpha p} \alpha p\left|\sin \frac{\pi \lambda_{v}}{\lambda_{n}}\right|^{\alpha p-1}\left|\sin \frac{\pi \lambda_{v}}{\lambda_{n}}-\sin \frac{\pi \lambda_{v-1}}{\lambda_{n}}\right| \\
& \leq \alpha p\left(\frac{2 \pi}{\lambda_{n}}\right)^{\alpha p} \lambda_{v}^{\alpha p-1}\left(\lambda_{v}-\lambda_{v-1}\right) .
\end{aligned}
$$

If $0<r<1$, then the similar estimate can be obtained using the inequality

$$
x^{r}-y^{r} \leq r y^{r-1}(x-y),
$$

which holds for all $x>0$ and $y>0[16$, Ch. 1].

Corollary 7. Suppose that the function $f \in B \mathcal{S}^{p}, 1 \leq p<\infty$, has the Fourier series of the form (3). Then for any $n \in \mathbb{N}$ and $\alpha>0$,

$$
\omega_{\alpha}^{p}\left(f, \frac{\pi}{\lambda_{n}}\right) \leq \alpha p\left(\frac{2 \pi}{\lambda_{n}}\right)^{\alpha p} \sum_{v=1}^{n} \lambda_{v}^{\alpha p-1}\left(\lambda_{v}-\lambda_{v-1}\right) E_{\lambda_{v}}^{p}(f) .
$$

If, in addition, the Fourier exponents $\lambda_{v}, v \in \mathbb{N}$, satisfy the condition

$$
\lambda_{v+1}-\lambda_{v} \leq C, \quad v=1,2, \ldots,
$$

with an absolute constant $C>0$, then

$$
\omega_{\alpha}^{p}\left(f, \frac{\pi}{\lambda_{n}}\right) \leq \frac{\alpha p(2 \pi)^{\alpha p}}{\lambda_{n}^{\alpha p}} C \sum_{v=1}^{n} \lambda_{v}^{\alpha p-1} E_{\lambda_{v}}^{p}(f) .
$$

\section{Constructive characteristics of the classes of functions defined by the generalized moduli of smoothness}

Let $\omega$ be the function (majorant) given on $[0,1]$. For a fixed $\alpha>0$, we set

$$
B \mathcal{S}^{p} H_{\alpha}^{\omega}=\left\{f \in B \mathcal{S}^{p}: \omega_{\alpha}(f, \delta)_{p}=\mathcal{O}(\omega(\delta)), \delta \rightarrow 0+\right\}
$$

Further, we consider the majorants $\omega(\delta), \delta \in[0,1]$, which satisfy the following conditions:

1) $\omega(\delta)$ is continuous on $[0,1]$;

2) $\omega(\delta) \uparrow$;

3) $\omega(\delta) \neq 0$ for $\delta \in(0,1]$;

4) $\omega(\delta) \rightarrow 0$ for $\delta \rightarrow 0$;

as well as the condition

$$
\sum_{v=1}^{n} \lambda_{v}^{s-1} \omega\left(\frac{1}{\lambda_{v}}\right)=\mathcal{O}\left[\lambda_{n}^{s} \omega\left(\frac{1}{\lambda_{n}}\right)\right]
$$

where $s>0$, and $\lambda_{v}, v \in \mathbb{N}$, is a increasing sequence of positive numbers. In the case where $\lambda_{v}=v$, the condition (40) is the known Bari condition $\left(\mathcal{B}_{s}\right)$ (see, e.g. [7]). 
Theorem 3. Assume that the function $f \in B \mathcal{S}^{p}, 1 \leq p<\infty$, has the Fourier series of the form (3), $\alpha>0$ and the majorant $\omega$, that satisfies the conditions 1)-4).

i) If $f \in B \mathcal{S}^{p} H_{\alpha}^{\omega}$, then the following relation

$$
E_{\lambda_{n}}(f)_{p}=\mathcal{O}\left[\omega\left(\frac{1}{\lambda_{n}}\right)\right]
$$

is true.

ii) If the numbers $\lambda_{v}, v \in \mathbb{N}$, satisfy condition (37) and the function $\omega^{p}$ satisfies condition (40) with $s=\alpha p$, then relation (41) yields the inclusion $f \in B \mathcal{S}^{p} H_{\alpha}^{\omega}$.

Proof. Let $f \in B \mathcal{S}^{p} H_{\alpha}^{\omega}$. Then relation (41) follows from (39) and (28).

On the other hand, if $f \in B \mathcal{S}^{p}$, the numbers $\lambda_{v}, v \in \mathbb{N}$, satisfy condition (37) and the function $\omega^{p}$ satisfies condition (40) with $s=\alpha p$ and relation (41) holds, then by (38), we get

$$
\omega_{\alpha}^{p}\left(f, \frac{1}{\lambda_{n}}\right)_{p} \leq \frac{C_{1}}{\lambda_{n}^{\alpha p}} \sum_{v=1}^{n} \lambda_{v}^{\alpha p-1} E_{\lambda_{v}}^{p}(f) \leq \frac{C_{1}}{\lambda_{n}^{\alpha p}} \sum_{v=1}^{n} \lambda_{v}^{\alpha p-1} \omega^{p}\left(\frac{1}{\lambda_{v}}\right)=\mathcal{O}\left[\omega^{p}\left(\frac{1}{\lambda_{n}}\right)\right],
$$

where $C_{1}=\alpha p(2 \pi)^{\alpha p} C$. Hence, the function $f$ belongs to the set $B \mathcal{S}^{p} H_{\alpha}^{\omega}$.

The function $t^{r}, 0<r \leq \alpha$, satisfies condition (40). Hence, denoting by $B \mathcal{S}^{p} H_{\alpha}^{r}$ the class $B \mathcal{S}^{p} H_{\alpha}^{\omega}$ for $\omega(t)=t^{r}$ we establish the following statement.

Corollary 8. Let $f \in B \mathcal{S}^{p}, 1 \leq p<\infty$, has the Fourier series of the form (3), $\alpha>0,0<r \leq \alpha / p$ and condition (37) holds. The function $f$ belongs to the set $B \mathcal{S}^{p} H_{\alpha}^{r}$, iff the following relation

$$
E_{\lambda_{n}}(f)_{p}=\mathcal{O}\left(\lambda_{n}^{-r}\right)
$$

is true.

In the spaces $\mathcal{S}^{p}$, for classical moduli of smoothness $\omega_{m}$, Theorems 2 and 3 were proved in [28] and [2]. In the spaces $\mathcal{S}^{p}$, inequalities of the form (38) were also obtained in [30]. In spaces $L_{p}$ of $2 \pi$-periodic functions, Lebesgue summable with the $p$ th degree, inequalities of the kind as (38) were obtained by M.F. Timan (see, e.g. [33, Ch. 6], [34, Ch. 2]). In the MusielakOrlicz type spaces, inequalities of the kind (32) were proved in [1].

\section{Acknowledgments}

This work is partially supported by the Volkswagen Foundation (VolkswagenStiftung), program "From Modeling and Analysis to Approximation".

\section{References}

[1] Abdullayev F., Chaichenko S., Shidlich A. Direct and inverse approximation theorems of functions in the MusielakOrlicz type spaces. Math. Inequal. Appl. 2021, 24 (2), 323-336. doi:10.7153/mia-2021-24-23

[2] Abdullayev F.G., Özkartepe P., Savchuk V.V., Shidlich A.L. Exact constants in direct and inverse approximation theorems for functions of several variables in the spaces $\mathcal{S}^{p}$. Filomat 2019, 33 (5), 1471-1484. doi:10.2298/fil1905471a

[3] Abdullayev F., Serdyuk A., Shidlich A. Widths of functional classes defined by majorants of generalized moduli of smoothness in the spaces $\mathcal{S}^{p}$. Ukrainian Math. J. 2021, 73 (6), 841-858. doi:10.1007/s11253-021-01963-6 (translation of Ukrain. Mat. Zh. 2021, 73 (6), 723-737. (in Ukrainian))

[4] Babenko A.G. On exact constant in the Jackson inequality in $L^{2}$. Math. Notes 1986, 39 (5), 355-363. doi: 10.1007/BF01156673 (translation of Mat. Zametki 1986, 39 (5), 651-664. (in Russian)) 
[5] Babenko V.F., Konareva S.V. Jackson-Stechkin-type inequalities for the approximation of elements of Hilbert spaces. Ukrainian Math. J. 2019, 70 (9), 1331-1344. doi:10.1007/s11253-019-01573-3 (translation of Ukrain. Mat. Zh. 2018, 70 (9), 1155-1165. (in Russian))

[6] Babenko V.F., Savela S.V. Inequalities of Jackson-Stechkin type for $B^{2}$-almost periodic functions. Visn. Dnipropetrovsk Univ. Ser. Math. 2012, 20 (6/1), 60-66. doi:10.15421/241208 (in Russian)

[7] Bari N.K., Stechkin S.B. Best approximations and differential properties of two conjugate functions. Trudy Moskov. Mat. Obshch. 1956, 5, 483-522. (in Russian)

[8] Bernstein S.N. On the best approximation of continuous functions by polynomials of given degree. In: Bernstein S.N. (Ed.) Collected Works, 1, Acad. Nauk SSSR, Moscow, 1952, 11-104. (in Russian)

[9] Besicovitch A.S. Almost periodic functions. Dover Publications, Inc., New York, 1955.

[10] Boman J. Equivalence of generalized moduli of continuity. Ark. Mat. 1980, 18 (1-2), 73-100. doi: $10.1007 /$ BF02384682

[11] Bredikhina E.A. Absolute convergence of Fourier series of almost periodic functions. Dokl. Akad. Nauk SSSR 1968, 179 (5), 1023-1026. (in Russian)

[12] Bredikhina E.A. Almost periodic functions. In: Vinogradov I.M. (Ed.) Encyclopedia of Mathematics, 4, Soviet Encyclopedia, Moscow, 1984, 543-545. (in Russian)

[13] Butzer P.L., Nessel R.J. Fourier Analysis and Approximation. Vol. 1. One-Dimensional Theory. Birkhäuser, Basel, 1971.

[14] Chernykh N.I. On the Jackson inequality in $L_{2}$. Proc. Steklov Inst. Math. 1967, 88, 75-78. (translation of Tr. Mat. Inst. Steklova 1962, 88, 71-74. (in Russian))

[15] Chernykh N.I. On the best approximation of periodic functions by trigonometric polynomials in $L_{2}$. Mat. Zametki 1967, 20 (3), 513-522. (in Russian)

[16] Hardy G.H., Littlewood J.E., Pólya G. Inequalities. Cambridge University Press, Cambridge, 1934.

[17] Horbachuk M.L., Hrushka Ya.I., Torba S.M. Direct and inverse theorems in the theory of approximation by the Ritz method. Ukrainian Math. J. 2005, 57 (5), 751-764. doi:10.1007/s11253-005-0225-4 (translation of Ukrain. Mat. Zh. 2005, 57 (5), 633-643. (in Ukrainian))

[18] Jackson D. Über die Genauigkeit der Annäherung stetiger Funktionen durch ganze rationale Funktionen gegebenen Grades und trigonometrische Summen gegebener Ordnung. Dissertation, Universität Göttingen, Druck der Dieterich'schen Univ.-Buchdruckerei W.Fr. Kaestner, Göttingen, 1911.

[19] Korneichuk N.P. On the exact constant in D. Jackson's theorem on best uniform approximation of continuous periodic functions. Sov. Math. Dokl. 1962, 3, 1040-1041 (translation of Dokl. Akad. Nauk SSSR 1962, 145, 514-515. (in Russian))

[20] Korneichuk N.P. Exact constants in approximation theory. Cambridge University Press, Cambridge, 2009.

[21] Kozko A.I., Rozhdestvenskii A.V. On Jackson's inequality for a generalized modulus of continuity in $L_{2}$. Sb. Math. 2004, 195 (8), 1073-1115. doi:10.1070/SM2004v195n08ABEH000838 (translation of Mat. Sb. 2004, 195 (8), 3-46. doi:10.4213/sm838 (in Russian))

[22] Levitan B.M. Almost periodic functions. Gosudarstv. Izdat. Tehn.-Teor. Lit., Moscow, 1953. (in Russian)

[23] Pritula Ya.G. Jackson's inequality for B²-almost periodic functions. Izv. Vyssh. Uchebn. Zaved. Mat. 1972, 8, 90-93. (in Russian)

[24] Pritula Ya.G., Yatsymirskyi M.M. Estimates of approximations of $B^{2}$-almost periodic functions. Visnyk of the Lviv Univ. Ser. Mech. Math. 1983, 21, 3-7. (in Ukrainian)

[25] Serdyuk A.S. Widths in the space $S^{p}$ of classes of functions that are determined by the moduli of continuity of their $\psi$-derivatives. In: Stepanets A.I. (Ed.) Extremal problems of theory of functions and related topics, Proc. Inst. Math. NAS Ukr., 2003, 46, 229-248. (in Ukrainian) 
[26] Serdyuk A.S., Shidlich A.L. Problems of approximation theory in abstract linear spaces. Zb. Pr. Inst. Mat. NAN Ukr. 2021, 18 (1), 594-643.

[27] Stepanets A.I. Approximation characteristics of the spaces $\mathcal{S}_{\varphi}^{p}$. Ukrainian Math. J. 2001, 53 (3), $446-475$. doi:10.1023/A:1012348506457 (translation of Ukrain. Mat. Zh. 2001, 53 (3), 392-416. (in Russian))

[28] Stepanets A.I., Serdyuk A.S. Direct and inverse theorems in the theory of the approximation of functions in the space $\mathcal{S}^{p}$. Ukrainian Math. J. 2002, 54 (1), 126-148. doi:10.1023/ A:1019701805228 (translation of Ukrain. Mat. Zh. 2002, 54 (1), 106-124. (in Russian))

[29] Stepanets A.I. Methods of approximation theory. VSP, Leiden-Boston, 2005.

[30] Sterlin M.D. Exact constants in inverse theorems of approximation theory. Sov. Math. Dokl. 1972, 13, $160-163$. (translation of Dokl. Akad. Nauk SSSR 1972, 202, 545-547. (in Russian))

[31] Taikov L.V. Inequalities containing best approximations and the modulus of continuity of functions in $L_{2}$. Math. Notes 1976, 20 (3), 797-800. doi:10.1007/BF01097254 (translation of Mat. Zametki 1976, 20 (3), 433-438. (in Russian))

[32] Taikov L.V. Structural and constructive characteristics of functions in $L_{2}$. Math. Notes 1979, 25 (2), $113-116$. doi:10.1007/BF01142721 (translation of Mat. Zametki 1979, 25 (2), 217-223. (in Russian))

[33] Timan A.F. Theory of approximation of functions of a real variable. Pergamon Press and MacMillan, Oxford, 1963. doi:10.1016/C2013-0-05307-8

[34] Timan M.F. Approximation and properties of periodic functions. Naukova Dumka, Kyiv, 2009. (in Russian)

[35] Vakarchuk S.B. Jackson-type inequalities and exact values of widths of classes of functions in the spaces $S^{p}$, $1 \leq p<\infty$. Ukrainian Math. J. 2004, 56 (5), 718-729. doi:10.1007/s11253-005-0070-5 (translation of Ukrain. Mat. Zh. 2004, 56 (5), 595-605. (in Russian))

[36] Vakarchuk S.B. Jackson-type inequalities with generalized modulus of continuity and exact values of the n-widths for the classes of $(\psi, \beta)$-differentiable functions in $L_{2}$. I. Ukrainian Math. J. 2016, 68 (6), 823-848. doi:10.1007/s11253016-1260-z (translation of Ukrain. Mat. Zh. 2016, 68 (6), 723-745. (in Russian))

[37] Vasil'ev S.N. The Jackson-Stechkin inequality in $L_{2}[-\pi, \pi]$. Proc. Steklov Inst. Math. 2001, 1, 243-253. (translation of Trudy Inst. Mat. i Mekh. UrO RAN 2001, 7 (1), 75-84. (in Russian))

[38] Voitsekhivs'kyj V.R. Jackson type inequalities in approximation of functions from the space $\mathcal{S}^{p}$. In: Stepanets A.I. (Ed.) Approximation theory and related topics, Proc. Inst. Math. NAS Ukr., 2002, 35, 33-46. (in Ukrainian)

Received 14.05.2021

Revised 15.11.2021

Сердюк А.С., Шилліч А.А. Прямі та обернені теореми наближення майже періодичних функиій у просторах Безиковича-Степания // Карпатські матем. публ. - 2021. — Т.13, №3. - С. 687-700.

$\triangle$ оведено прямі та обернені теореми наближення у просторах Безиковича-Степанця $B \mathcal{S}^{p}$ майже періодичних функцій в термінах найкраших наближень функцій та їх узагальнених модулів гладкості.

Ключові слова і фрази: пряма апроксимаційна теорема, обернена апроксимаційна теорема, нерівність типу Ажексона, узагальнений модуль гладкості. 